\title{
Acceptability of driving an equipped vehicle with drive recorder: the impact of the context
}

ISSN 1751-956X

Received on 22nd July 2014 Revised on 13th April 2014 Accepted on 26th May 2015 doi: 10.1049/iet-its.2014.0174 www.ietdl.org

10

\author{
Chloé Eyssartier $\bowtie$ \\ DTEROUEST, CEREMA, Dimer, Man Rue René Viviani, BP 46223-44262 Nantes Cedex 2, 44626 Nantes Cedex 2, France \\ $\bowtie$ E-mail: chloe.eyssartier@cerema.fr
}

Q1 Abstract: The objective of this study conducted in the S_VRAI project [saving lives by road incident analysis feedback] is to present results about the acceptability of equipping a vehicle with an event data recorder (EDR) without feedback. Five focus groups were conducted in two different French Civil Service departments. Lack of privacy, volunteering, anonymity and the data access are main elements of the acceptability of the EDR. The results show also the importance of the societal and professional context when studying the acceptability of an EDR. However, all these elements need to be moderated by the corporate culture.

The S_VRAI project [saving lives by road incident analysis feedback], financed by the French government, Road Traffic and Safety Directorate aims to equip fleets of vehicles with drive recorders (event data recorder (EDR)) in order to collect incidents [near accidents] for road safety purposes. In other words, an incident is defined as dangerous driving situations in which the driver has no control or nearly no control and that does not always cause physical damage [1]. More technically information can be found in Serre et al. [2]. The objective of the project is to have a better idea of the

81 causes of dangerous driving situations. It is managed by IFSTTAR and CEREMA. About 54 vehicles were equipped with an EDR called EMMA that records data (speed, acceleration, echolocation data, ...) before and after an incident occurs.

However, no feedback on driving behaviour is being done at this stage, because the CNIL [National Commission on support and 40 innovative and personal liberties], that protects the privacy of French people has not given its agreement to make the link between a specific driver and his driving behaviour. For this reason, the data needs to be anonimysed. The drivers have all volunteered to participate in the project and the data is rendered 45 anonymous before being analysed.

If studying the technical aspect of a new tool is essential, studying its acceptability is also relevant. As already mentioned by Mitsopoulos et al. [3, p. 439] about ITS technologies 'in order for in-vehicle ITS technologies to be successful in reducing the incidence and severity of road crashes, the technologies must be

50 deemed to be acceptable by eventual road users [..] ITS technologies that are not acceptable to drivers are unlikely to have the desired effect on driver behaviour'. In fact, an automated system that is efficient but not acceptable will not be adopted by the users. Therefore, one of the secondary objectives of the project

55 is to study the acceptability of equipping vehicles with the EDR-EMMA. In France, vehicles are not equipped with an EDR unlike in several foreign countries. In this paper, we are focusing on acceptability because the participants have not yet driven an equipped vehicle.

Few works $[4,5]$ have focused on the acceptability of an EDR 60 without feedback on driving behaviour, which gives this paper its innovative aspect. In all the documents an EDR is a tool that records driving behaviours such as speed and acceleration, it does not only focus on accident situations but on dangerous ones. In this paper, we will focus on the acceptability of the system itself 65 and not on the impact of the acceptability on driving behaviours.
The interest of this paper is first that a few researches were made on the acceptability of an EDR without feedback and no research was made in France on this topic although it is likely that individuals' acceptability of technology is influenced by the cultural climate in which the technology is introduced. Second, research is required to evaluate driver acceptability before EDR deployment on a larger scale. It is counterproductive to invest time and effort in designing and building equipped vehicle if the vehicles are not purchased by the drivers or if the EDRs in the purchased vehicles are disabled. In this way, elements of the technology deemed unacceptable by users can be rectified prior to system.

\subsection{Lack of privacy}

The lack of privacy is the main element in the non-acceptability of the EDR $[4,5]$. Past research has focused more on the acceptability of the EDR with feedback on young drivers, on professional drivers and on private drivers:

- Parents-children: Results of the literature show that some parents, even if they feel concerned by their children's road behaviour, do not agree to have access to their driving behavioural data in that it is an attack on privacy and could have a negative impact on the relationship of trust they have established with their children [6]. They consider it could remove responsibility from their children, who have the right to make their own mistakes [7]. However, other parents appreciate, knowing about their children's risky behaviour such as speeding or drink driving, especially during the probationary driving period.

Therefore, for the reasons mentioned above but also because of the difficulty of using new technologies, only half of the parents know how to use the web site where the behavioural data are recorded [8].

As for the children themselves, they do not like the idea that their behaviour could be recorded in real time and that their journeys could be monitored [6]. For them, the interest of their parents in this kind of information shows a lack of trust [6].However, the results show a positive impact of this method on driving behaviour for high-risk drivers [9].

- Professional drivers: Concerning professional drivers, a lot of research has been conducted on the impact of this system on accidents or on driving behaviour ['salesman' [10]; 'ambulance 
drivers' [11-14]]. However, a few works have been conducted on its acceptability while 'the most important challenge in applying on-board safety monitoring to commercial motor vehicle driver safety management is likely to be achieving driver acceptance' $[15$, p. 29]. Therefore, according to some works, for professional drivers, there is an increase in their perceived stress [16], this technology represents an attack on privacy [15-17] that could have a negative effect on the driver's judgment and modify his driving behaviour [14]. On this point, so as not to worry the drivers, some researchers propose that the drivers, and not their manager, should be the only ones that can have access to their own data and to their analysis [15].

- Private drivers: The lack of privacy concerns private drivers too. Nearly 400 American drivers, on 790 respondents (more than half of the respondents), say that the EDR threatens their privacy and especially if its use is compulsory [4]. This threat is mentioned also in a French research study, about new technologies in the vehicles 'the aspect of a strong feeling of control is often mentioned (fear of the black box)' [18, p. 7].

When drivers have to give their opinion on several systems: the EDR and systems that get people to respect the speed limits [the intelligent speed adapter and the section control], it is the EDR that seems to have the strongest impact on privacy. The authors of the research quoted above conclude that 'the measures will be acceptable if they are perceived as efficient and fair. On the other hand, the acceptability will be weakest if the measures are perceived as a lack of privacy' $[5, \mathrm{p}$. vii]. That is the reason why the eCall [automatic crash notification] is one the most acceptable systems because of its obvious safety benefits in emergency situations, and because it does not interfere with basic driving habits $[19,20]$. Moreover, it is perceived as useful especially in rural areas [21].

\subsection{Data access}

As seen before in this paper, the data access and their use are two of the main aspects of the lack of privacy. Therefore, the question remains by whom and for what purpose the data can be used.

The concern that the data can be stolen is an illustration of the importance of data access.

Therefore, according to American drivers in two research studies, insurance companies should have access to the data recorded by the EDR to know the causes of the accident but also to help them to define the total amount of insurance that the drivers would have to pay $[22,4]$.

The other two entities that should have access to them are the police and the legal profession [4]. In case of accidents, the EDR is a neutral element that gives objective information [22] even if it could be a specific charge if the driver is responsible for the accident [22]. The recorded data could be useful for car manufacturers to improve the safety of vehicles $[4,22]$. Finally, the driver himself would like to have his own data [4] as a means to improve his driving behaviour. The data could even be helpful in finding a job in an area where driving behaviour is a main element of the job (e.g. professional driver) [23].

\subsection{Volunteering}

Even if voluntary use is often quoted (unified theory of acceptance and use of technology [24]; technology acceptance model [25]; and innovation diffusion theory [26]), it is not studied in the research on the acceptability of the advanced driver assistance systems.

This is perhaps because using new technologies is based on individual choice.

As for the acceptability of EDR, we can make the same comment, voluntary use is not studied very often. As we saw in the section on privacy, EDR is used in a professional context as a way to improve professional drivers' behaviour. It is an obligatory tool and so voluntary use is not relevant.
According to the commitment theory [27], freedom is a necessary condition to reinforce attitudes (positive attitudes about the project), doing and persevering in the expected behaviour (no retraction or circumvention and avoidance strategies) [28]. Therefore, giving people free choice to drive an equipped vehicle could keep positive attitudes stronger, increasing the probability that they will take part and persevere. That is why an obligatory tool with a low acceptability will generate circumvention and avoidance strategies.

\subsection{Social context}

It would be wrong to consider that the acceptability of a tool is a unique relation between the tool and its user without taking into account the social context (colleagues, friends, societal organisation, ...).

The elements quoted in the international literature do not take into account the social or professional context in which the EDR is used. However, the importance of the psychological elements that allow a better understanding of the acceptability is defended by several authors $[29,30]$.

Therefore, according to the explanatory levels [31], inter-personal relations are important in terms of the study of social influence. The social norm influence is a good illustration of this analysis level. Some authors [32] distinguish two types of norm: a subjective descriptive norm ('what the others do') and an injunctive subjective norm ("what I believe the others expect me to do'). In other words, a tool will be more acceptable if others use it, as is the case for the parents that agree more to use an EDR with feedback on behaviour if other parents do the same thing [7].

\section{Objectives}

The objective of this paper is to study the acceptability of the EDR before starting to use it with civil servants. We based the study on the literature on the subject and we made the choice to focus also on other aspects such as professional context and personal context. As it is an explanatory research, we do not have a precise hypothesis but we suppose that the social context will play a role in the acceptability of driving a vehicle equipped with an EDR.

We are interested in the acceptability of the EDR by the people willing to drive an equipped vehicle but also, to know the barriers to the acceptability of the EDR-EMMA, we are interested in the people unwilling to drive an equipped vehicle.

\section{Method}

To study the acceptability of driving a vehicle equipped with an EDR, we choose to make focus groups. The focus group method can provide information on an unknown subject. This qualitative method has already been used by several researchers in relation to the acceptability of the EDR [4] but also concerning the acceptability of new technologies [33].

\subsection{Procedure}

Two different bodies of the French civil service agreed to equip some of their vehicles with drive recorders. The first one (called A) is a government entity and the second one (called B) a local authority. More precisely, the first one liaises between the government and the local structures at a regional level in that it helps, if needed, the local ones to implement public policies. The second ones work only at a local level (on a smaller area) and their work is to improve the quality of life (safety, infrastructure, ...) of the inhabitants of the county.

The focus group met during working hours and the participants gave a written consent for their discussion to be audio-recorded.

About 28 civil servants participated in this paper: 22 in the entity A, with 4 not willing and 18 willing to drive an equipped vehicle and 6 willing to drive an equipped vehicle in the entity B (see Table 1). 
Table 1 Composition of the focus groups

\begin{tabular}{|c|c|c|c|c|c|}
\hline \multicolumn{4}{|l|}{ Entity A } & \multicolumn{2}{|c|}{ Entity B } \\
\hline \multirow{2}{*}{$\begin{array}{l}\text { Not willing } \\
\text { Employees }\end{array}$} & \multicolumn{3}{|c|}{ Willing } & \multicolumn{2}{|c|}{ Willing } \\
\hline & Empl & Dyees & Managers & Employees & Head manager \\
\hline FG & FG & FG & $\mathrm{FG}$ & $\mathrm{FG}$ & $\begin{array}{l}\text { Individual } \\
\text { interview }\end{array}$ \\
\hline$N=4$ & $N=5$ & $N=7$ & $N=6$ & $N=5$ & $N=1$ \\
\hline
\end{tabular}

Groups were intentionally structured to be homogenous in nature to encourage discussion amongst people at the same level in the hierarchy. Therefore, the managers were not mixed with their 280 employees. That is why in the entity $\mathrm{B}$, an individual interview on the same themes was conducted with the head manager while one focus group was made with the employees.

\subsection{Material}

Participants were informed that all opinions were valued equally and that divergent views were welcome. A semi-structured interview format using open-ended questions was used, providing the opportunity for exploration on concepts of interest, as well as for

290 free-flowing conversation amongst participants and expansion of ideas within group conversation. Questions were designed to highlight elements from the literature, beginning with a specific subject (the EDR) and finishing with more general ones (generalisation of the EDR). More precisely, the semi-structured

295 interview format was composed of four themes: the knowledge of the project, the EDR-EMMA (its reliability, the data access, its usefulness, the lack of privacy and the importance of the voluntary aspect), the social environment (colleagues' and relatives' views about the project, the perceived impact on driving behaviour, the impact of the project on working life in general) and the generalisation of the EDR (willingness to equip personal vehicles).

\subsection{Analysis}

305 The author facilitated each group (50-85 min duration) and a private company transcribed the discussion verbatim.

The software programmes [34] used to analyse individual interviews such as Spad-T, Sphynx, Tropes and Alceste do not seem useful for focus groups, which are not structured enough. During a focus group, the interaction between the respondents bring them to speak about several subjects not necessary linked directly to the asked question that is why we consider that focus groups are not structured enough comparatively to individual interviews, for example. Other software known as computer-Aided qualitative data analysis systems is more relevant. However it is

315 not used very often in France, which is why the researchers need to learn how to use it in foreign countries.

As a consequence, the thematic analysis seems more relevant on this paper. Thematic analysis using an interpretative framework was used [35]. This is done by scrutinising participant responses to each question-topic area to identify themes that are common across groups as well as those that are unique to particular groups. Overall, transcripts were analysed keeping the literature elements in mind while remaining as open as possible to emerging themes that did not fit within the theoretical framework.

\section{Results}

First, we will present the results about common elements between 330 the two entities, and second the differences between them.
As mentioned at the beginning of this paper, all the participants volunteered to participate in the focus group and all the data were rendered anonymous before analysis.

\subsection{Main common elements between the two entities}

The common elements are lack of privacy and data access.

- Lack of privacy: Some respondents highlight that the lack of privacy is not relevant in the area of road safety because the road is a shared space. Therefore, for the safety of most drivers, it could be useful to decrease individual privacy. Therefore, it is a question of knowing if the collective interest, (increasing the road safety) should be more important than the private (having a system in the car that is perceived as a lack of privacy that will record driver behaviour) or, more precisely, at which moment the collective interest comes first.

The respondents do not think that this system could have an impact on their privacy insofar as they have to drive a professional vehicle. However, the participants do not agree to fit their private vehicle, considering that it is an invasion of privacy. There are opposing views for private vehicles and professional vehicles. That means that they agree to a minimum of monitoring in their professional context.

As for equipping a private vehicle, the other contentious points in addition to the invasion of privacy are the cost of such a tool and its effectiveness. More precisely, the cost should not be higher than $100 €$ and such a tool should have a positive impact on the number of accidents and modify the behaviour of unsafe drivers.

- Data access: For the respondents, some end-users should have access to the data and other should not have.

Two end-users could have access to the data: the drivers and the car manufacturers. Data access by car manufacturers is justified because it would allow to increase the safety of the vehicle. Similarly, road users would like to access to their own driving behavioural data. However, the respondents query the format of the data that could be sent to them, and especially about their understanding of it. In conclusion, they would like to have access to a summary of the results. The respondents wonder about the possibility of having access to this data in the case of litigation with another road user. The objective data could be helpful if there is an accident or if the respondents want to contest an offense. However, it could be a specific charge for the driver.

One of the doubts is about the data used by the hierarchy. If the participants know that the data will not be sent to management during the project, they worry about what could happen at the end of the project if management asks to have access to it. According to them, the data that could interest management is that which allows them to know where the employees are and in the case of an accident, if the employees are responsible.

Concerning the insurance companies, most of the respondents do not think that they would have access to the data as, according to them, the objective of these companies is to make profits.

If this option is chosen, the participants would like insurance premiums to decrease even if others worry about an increase.

\subsection{Differences between the two entities}

The differences concern volunteering, anonymity, social support and professional and societal environment:

- Volunteering: Concerning the importance of the voluntary nature of the action, we can observe differences between the respondents in entity A and entity B. Therefore, for the respondents of the entity A, voluntary action is a non-negotiable element of the acceptability to be involved in the project. Compulsory participation could justify a refusal to get involved. If they have to be involved, if they do 
not have the choice, they will find a way to skew the data (to trigger the EDR...). However, for the respondents of the second entity, volunteering is not important in a professional context, since they consider that they have to apply the rules issued by the management of their entity.

The respondents that did not volunteer to drive an equipped vehicle (who are all in the entity A) speak about manipulation. According to them, it would create the appearance that the management needs to have their agreement however, the decision has already been taken to involve the entity in the project. In other words for them, if no respondents agreed to be involved in the project, the manager would impose the obligation to drive an equipped vehicle. The goal would be to avoid objections and to unite around the project. However, according to them, this way of thinking targets the general policy of the entity and not only about the project management.

- Anonymity: Generally, the respondents trust in the procedure even if they speak about bypass strategies (read the erased files, know the driver identity in cross-checking the date of the file, the planning system for car...). The fact that the legal profession can truly have access to the data in the case of accidents makes people think that it is possible to know the identity of the drivers. In other words, the anonymisation process can be circumvented. The question of anonymisation is also mentioned in relation to the video data. This kind of data is not recorded in the S_VRAI project but the point was nonetheless discussed during the focus groups. It is not the fact to record the driving behaviour from data such as speed that is perceived as an invasion of privacy but to film the driver.

Similarly, it is over the interest of anonymising the data that the opinions are divided. For the respondents of entity A, data anonymity is an essential element of their willingness to be involved in the project while it is not the case for the respondents of entity B.

- Social support: Social influence is a major element in the acceptability of new technologies. In this project, it is about personal and professional support.

Their participation in the project has not been mentioned with their line-management. This was confirmed by the managers of entity A, who specified that they did not speak about the project with their employees. At the opposite, in the entity B, the managers do not speak about the project with their employees because they do not need it. They are the only people that drive an equipped vehicle. Contrary to the entity $\mathrm{A}$, each vehicle is not shared with the other drivers; it is driven by only one driver. Whether the entity A or the entity B, there was also no discussion with their colleagues about the project. Concerning family, most of the participants have not spoken with them about their participation in the project. The few people that spoke about this with family and with their colleagues say that they tease them not nasty.Therefore, one of the reason about the lack of discussion between the participants and their colleagues and relatives could be that they do not worry about being involved in this project.

- Professional and societal environment: This section is about the social and professional context in which people live and its impact on their behaviour. We did not expect to include these results but it is the discussion between the respondents during the focus groups that caused these elements to emerge. Therefore, the general rules of the entity where the respondent works, the families' experiences or changes in society will have an impact on the acceptability of the EDR.

oProfessional environment: The EDR acceptability depends on the context of use, here the professional context.

In entity A, the project is implemented at the same time as efforts to prevent abusive professional practices. Only those not willing to participate in the project say that the reason they refuse is because it is another way to monitor their behaviour. This aspect is raised by the volunteers of entity A that point out that their journeys are more heavily monitored but they adapt by informing their hierarchy when they slightly bend the rules. In other words, these measures are not perceived as very restrictive by these respondents.

Another point is about the implementation of the project which was done at the same time as the installation of global positioning system in a fleet of vehicles in a different workplace. In each focus group, this event was mentioned. For this entity, the objective was to be more efficient in knowing where the employees were, so that they could tell them to go to a specific destination if needed. The employees did not agree to such a measure, as they had the feeling of being monitored so they protested and even held their manager hostage. However for the participants, this case has nothing in common with the S_VRAI project, in which the data is rendered anonymous.

In the entity $\mathrm{B}$, none of these aspects were evoked by the respondents even if the activities are quite the same. The discussion was only about the S_VRAI project:

o Societal aspect: The institutional aspect in the country where the automated systems are fitted has to be taken into account when we make an acceptability study. Therefore in France, the CNIL [National Commission on support innovative and personal liberties] plays a protective role in the privacy of French people. Its objective is 'to protect personal data, support innovation and preserve individual liberties' [36]. In the two entities, the respondents trust the CNIL and it is an important element of their acceptability. For them if the CNIL gives its agreement to the project to be implemented, it is a gage of reliability. Their privacy will be respected and that their data will not be sent to insurance companies. The respondents that did not want to be involved in the project do not speak about the CNIL at all. As this element was never mentioned in the literature and that these respondents were the first ones met by the researcher, the question was not asked.

In a completely different field, the impact of wireless fidelity (wifi) waves on health was discussed in the media in France, so the respondents were worried by the fact that the data will be sent by wifi from the EDR fitted in the car to the entity that will analyse the data. This worry is not mentioned very often but it illustrates the interest of focusing on the social context and on the themes related to the subject studied.

\section{Discussion}

This final part will present the main results of this paper, its limits and the research opportunities.

\subsection{Conclusion}

The acceptability of an EDR is not an exclusive relation between the user and the system but it needs to take into account the social organisation in which the user is situated.

It is essentially based on data access and its use. The respondents agree that the car manufacturers and the drivers should have access to their own data. They have more doubt concerning the legal system and are against management or insurance companies having access to this information. This last result is not consistent with the literature, where the participants think that the data can be useful for insurance companies to define the amounts of insurance and the accident causes. In France, there is a certain distrust of insurance companies [4, 22].

The main finding of the work is about the importance of the context. Some main differences appeared on some factors probably due to organisational differences. Therefore, between the respondents of entity A and those of entity B, the frontier is not the same. The first ones accept less interference from their managers in their work than those of entity B. The voluntary

465 
nature and the data anonymity are perceived by the respondents of 530 the entity A as necessary elements in their willingness to be involved in the project, whereas it is perceived as optional for the respondents of entity B. Similarly, the respondents of entity B, who drive a regular car, do not have a stronger feeling of lack of privacy than the respondents of entity A, who drive a car from a shared fleet.

535 Several explanations could be proposed to explain these results. One of them is that the sample in the entity B is too small (six people) comparatively to the one of the entity A (22 people). According to the small numbers of respondents in the entity B, the impact of the personal characteristics on the results could not be ignored.

In line with some authors [30], who write that the organisational context is an explanatory factor of the acceptability, we show in this paper that the professional context has an impact on the acceptability. Moreover, some researchers [30] add another element concerning social organisation. 'This organisational level 545 will allow to take into account the specific context in which the individual is' [30, p. 391]. Therefore, we could suppose that the acceptability of a tool, in a professional context, is dependent on the organisation of the company but also on the corporate culture. This finally is defined as a 'global vision of the organisation and 50 its objective' [37].

A rich literature exists about the different ways of running in terms of management (see 37 for summary). Therefore, the observed differences are based either on the contractualised rules or on differences in terms of the corporate culture. In a complementary way to the concrete rules in the firm (employment contract, ...),

555 the corporate culture plays a role of 'organisational coordination' [38, p. 179].

'By dint of working together, the agents make rules, values and common accepted practices to manage solidarity and self-helps, technical complementarity and authority, trainings and information and control and evaluation [...]. The firm culture germanes to an organisational common sense, to a package of collective believes internalised by the agents' $[38$, p. 179].

In other terms, are the differences between the agents of entities A and $\mathrm{B}$ due to contractualised rules or/and to informal and implicit rules that the agents implement and take on board? Probably both, however as things stand, it is impossible for us to provide a clear explanation on this difference between the two entities. To clarify this point, a specific study on this aspect needs to be implemented. The elements that we know about the missions of each entity

570 (more operational and more local for the entity B) and the organisation of the different entities (that are quite the same, structured in thematic group) could not bring explanations for this result.

However in a more general way, the professional environment could have an impact on the acceptability of the EDR. Therefore, as the implementation of the project is made at the same time as efforts to prevent abusive professional practices, for the non-volunteer of entity A, the EDR is another way to monitor their behaviour.

To the explanatory levels developed by Doise [31] and completed

580 by Terrade et al. [30], we would add another one, the society context. This idea is quoted in other French works about new technologies, the researchers highlight that the people they met think of ' $a$ strong feeling of a control paradigm added with a societal context, a feeling that French government control everyone' [18, p. 7]. Therefore, the CNIL is a French entity that protects individual liberty. For the participants, if the CNIL gives its consent to the S_VRAI project, it is an assurance that their privacy will be respected. We can see that the society organisation has an impact on the acceptability.

\subsection{Limitations}

We could regret that so few non-volunteer participants to drive an equipped vehicle agreed to be involved in this paper. Obviously, the fact that the meeting took place during working hours and that these hours were considered by the line managers as real work were not enough of an incentive to convince them to be involved in the focus groups.

Similarly, it would be interesting to meet more people in entity B that are willing to be involved in the project but also people who are not willing. That would make it possible to validate the hypothesis about the differences between entities with a larger sample.

\subsection{Research opportunities}

First, a second phase of focus group is conducted to evaluate the acceptance of driving an equipped vehicle after having driven such a vehicle. The idea is to compare the results of the acceptability and the acceptance. The data are currently being analysed.

Later, the project will target the installation of an EDR in 500 vehicles, so according to the results of this paper, volunteering, data access and anonymisation will be the main elements of the acceptability. However, these elements will need to be moderated according to the type of entity (entity A; entity B). In this second phase, the vehicles will be equipped with a video to have more information on the causes of an incident. The results of the research presented here show that filming the road is not an issue while filming the driver is perceived as an invasion of privacy. Therefore, to promote the acceptability of this new version of the EDR-EMMA (with a video), it will be better to film the road instead of the driver.

Insofar as an explanation of the results could be that the corporate culture has an impact on the acceptability of the EDR, we could test this hypothesis on several entities (private versus public, national group versus local ones, ...), to define the differences in terms of corporate culture between the entities and why these differences have an impact on the acceptability to drive a vehicle equipped with an EDR.

\subsection{Road safety applications}

Moreover, this paper presents measures to improve the acceptability of equipping private vehicle with an EDR. Therefore, the cost and the effectiveness are elements that we need to take into account. However, it is possible that once the vehicles will be equipped with an EDR, other factors will appear that were not mentioned in the acceptability study.

However before considering such a measure, a preliminary study on this precise aspect seems essential. The objective of the research presented in this paper was not to study the acceptability of equipping its own vehicle with an EDR by the way the elements that we have on this aspect do not let us to have a global vision of this question.

An other possibility should be considering to equip professional vehicles in taking into account the characteristics of the different structures in terms of corporate culture. On the basis of a research that needs to be conducted, a firm classification could be made according to the corporate culture but mainly of its impact on the acceptability, we will then be able to propose specific attempts to insure a good acceptability of the EDR.

To conclude, this paper is the first one made in France on the acceptability of an EDR. Our objective is to show that the acceptability of an EDR is much more complex than a relation between the system and its user even if it is what is done for most of the research on the acceptability of new technologies in road safety. To appreciate all the complexity of the acceptability, it can be assumed to take into account the social, professional, personal and societal context.

\section{References}

1 Guilbot, M.: Rapport sur les aspects juridiques. Le projet S_VRAI et la protection des données à caractère personnel. Dossier de présentation pour la demande d'autorisation à la CNIL concernant la phase 1 du projet. Convention de Subvention DSCR/Certu/INRETS n7624 (2011)
595

600 
2 Serre, T., Naude, C., Chauvet, S., Fournier, J.Y., Lechner, D., Ledoux, V.: 'Towards a classification of road incidents acquired from public fleets of vehicles'. Second Int. Symp. on Future Active Safety Technology towards Zero-traffic Accident, Nagoya, Japan, September 2013

3 Mitsopoulos, E., Regan, M.A., Haworth, N.: 'Acceptability of in-vehicle intelligent transport systems to Victorian car drivers'. Australian Road Safety Conf., Gold Coast, October, 2002

4 Gabler, H.C., Gabauer, D.J., Newell, H.L., O'Neill, M.E.: 'Use of event data recorder (EDR) technology for highway crash data analysis'. NCHRP, 2013

5 Bjørskau, T., Assum, T., Eriksson, L., Hrelja, R., Nyberg, J.: 'Summary: privacy protection and ITS-based road safety measures'. TØI Report, 1097, 2010

6 Guttman, N., Gesser-Edelsburg, A.: 'The little squealer' or 'the virtual guardian angel'? Young drivers' and their parents' perspective on using a driver monitoring technology and its implications for parent-young driver communication', J. Saf. Res., 2011, 42, pp. 51-59

7 Guttman, N., Lotan, T.: 'Spying or steering? Views of parents of young novice drivers on the use and ethics of driver-monitoring technologies', Accident Anal. Prev., 2011, 43, pp. 412-420

Prato, C.G., Toledo, T., Lotan, T., Taubman-Ben-Ari, O.: 'Modeling the behavior of novice drivers during the first year licensure', Accident Anal. Prev., 2010, 42, (2), pp. $480-486$

9 Mc Gehee, D.V., Raby, M., Carney, C., Lee, J.D., Reyes, M.: 'Extending parental monitoring using event-triggered video intervention in rural teen drivers', J. Saf. Res., 2007, 38, pp. 215-227

10 Wouters, P.J., Bos, J.M.: 'Traffic accident reduction by monitoring driver behaviour with in-car data recorders', Accident Anal. Prev., 2000, 32, pp. 643-650

11 Levick, N., Wiersch, L., Nagel, M.E.: 'Real World Application of an Aftermarket Driver Human Factors Real-Time Auditory Monitoring and Feedback Device: An Emergency Service Perspective'. Paper Number 07-0254, 2004

12 Lehman, G., Cheale, A.: 'The Contribution of Onboard Recording Systems to Road Safety and Accident Analysis'. Paper Number 98-S2-0-34, 2005

13 Lewick, N.R., Swanson, J. 'An optimal solution for enhancing ambulance safety implementing a driver performance feedback and monitoring device in ground emergency medical service vehicle'. Proc. 49th Annual Proc., Association for Advancement of Automotive Medicine, September 2005

14 Hickman, J.S., Hanowskia, R.J.: 'Use of a video monitoring approach to reduce at-risk driving behaviors in commercial vehicle operations', Transp. Res. F, Traffic Psychol. Behav., 2011, 14, (3), pp. 189-198

15 Knipling, R.R., Hickman, J.S., Bergoffen, G.: 'Commercial truck and bus safety synthesis program (U.S.)' (Federal Motor Carrier Safety Administration, USA, 2003)

16 Horrey, W.J., Lesche, M., Dainoff, M., Robertson, M., Noy, L.: 'On-board safety monitoring systems for driving: review, knowledge gaps and framework', J. Saf. Res., 2012, 43, (2), pp. 49-58

17 Huang, Y.H., Roetting, M., Mc Devin, J.R., Melton, D., Smith, G.S.: 'Feedback by technology: attitudes and opinions of truck drivers', Transp. Res. F, 2005, pp. $277-297$
18 Ralf, E., Krishnakumar, R., Langlois, A.: 'Acceptabilité des nouvelles technologies dans l'automobile'. Proc. of the 17th Canadian Multidisciplinary Road Safety Conf., Québec, June 2007

19 Beanland, V., Lenné, M.G., Fuessl, E., et al.: 'Acceptability of rider systems for powered two-wheelers', Transp. Res. F, 19, pp. 63-76

20 Beanland, V., Lenné, M.G.: 'An international study of the factors associated with the acceptability of advanced rider assistive systems for powered two-wheelers'. Proc. of the 2013 Australasian Road Safety Research, Policing \& Education Conf., Brisbane, Queensland, 28th August 2013

21 Cairney, P.: 'Consumers' views of information technology based systems for passenger cars (RR ARR 277)' (ARRB Transportation Research, 1995)

22 Athavale, M., Borna, S.: 'Some issues for insurers on the use of event data recorders', J. Insurers Issue, 2008, 31, (1), pp. 1-15

23 ROSPA: 'Road safety and in-vehicle monitoring technology'. (http://www.rospa. com Birmingham, 2013)

24 Venkatesh, V., Morris, M.G., Davis, G.B., Davis, F.D.: 'User acceptance of information technology: toward a unified view', MIS Q., 2003, 27, (3), pp. $425-478$

25 Nielsen, J.: 'Usability engineering' (Academic Press, 1993)

26 Rogers, E.: 'Diffusion of innovation' (Free Press, 1995)

27 Kiesler, C.A.: 'The psychology of commitment. Experiments liking behavior to belief' (Academic Press, 1971)

28 Joule, R.V., Beauvois, J.L.: 'Petit traité de manipulation à l'usage des honnêtes gens' (P.U.G, 2002)

29 Bobillier-Chaumont, M.E., Dubois, M.: 'L'adoption des technologies en situation professionnelle; quelles articulations possibles entre acceptabilité et acceptation ?', Le Trav Humain, 2009, 72, (4), pp. 355-382

30 Terrade, F., Pasquier, H., Reerinck-Boulanger, J., Guigouain, G., Somat, A.: 'L'acceptabilité sociale: la prise en compte des déterminants sociaux dans l'analyse de l'acceptabilité des systèmes technologiques', Le Trav. Humain, 2009, 72, (4), pp. 383-395

31 Doise, W.: 'L'explication en psychologie sociale' (Presses universitaires de France, 1982)

32 Kallgren, C.A., Reno, R.R., Cialdini, R.B.: 'A focus theory of normative conduct: when norms do and do not affect behaviour', Personality Social Psychol. Bull., 2000, 26, pp. 1002-1012

33 Regan, M.A., Mitsopoulos, E., Haworth, N., Young, K.: 'Acceptability of in-vehicle intelligent transport systems to Victorian car drivers'. Royal Automobile Club of Victoria Research Report RACV, 2002

34 Duchesne, S., Haegel, F.: 'L'enquête et ses méthodes: l'entretien collectif' (Nathan, coll. 128, 2008)

35 Templeton, J.A.: 'The focus group, revised edition' (McGraw-Hill, 1994)

36 CNIL, http://www.cnil.fr/english/, accessed July 2014

37 Cohendet, P., Diani, M.: 'L'organisation comme une communauté de communautés: croyances collectives et culture d'entreprise', Rev. d'Économie Politique, 2005, 113, (5), pp. 697-721

38 Diani, M.: 'Économie Evolutionniste et culture d'entreprise'. PhD thesis, Université Louis Pasteur, Strasbourg, 2003 


\section{ITSSI20140174}

795 Author Queries

Chloé Eyssartier

Q1 Please provide the expansion of the acronyms VRAI, IFSTTAR, CEREMA, EMMA, CNIL and ITS.

Q2 Please provide volume number for Ref. [17].

Please provide publishing year for Ref. [19].

Q4 Please provide the publisher location in Refs. [25-28, 34, 35]. 